\title{
Resveratrol can enhance osteogenic differentiation and mitochondrial biogenesis from human periosteum-derived mesenchymal stem cells
}

\section{Dong Kyu Moon}

Gyeongsang National University Hospital

\section{Bo Gyu Kim}

Gyeongsang National University

A Ram Lee
Gyeongsang National University

\section{Yeong In Choe}

Gyeongsang National University

Imran Khan New

Gyeongsang National University

\section{Kyoung Mi Moon New}

Gyeongsang National University

\section{Ryoung-Hoon Jeon}

Gyeongsang National University

\section{June-Ho Byun}

Gyeongsang National University Hospital

\section{Sun-Chul Hwang}

Gyeongsang National University Hospital

Dong Kyun Woo ( $\square$ dongkyun.woo@gnu.ac.kr)

Gyeongsang National University

\section{Research article}

Keywords: Mesenchymal stem cell, Resveratrol, Osteogenesis, Mitochondria

Posted Date: April 6th, 2020

DOI: https://doi.org/10.21203/rs.2.21688/v2

License: (1) (1) This work is licensed under a Creative Commons Attribution 4.0 International License.

Read Full License 
Version of Record: A version of this preprint was published at Journal of Orthopaedic Surgery and Research on June 3rd, 2020. See the published version at https://doi.org/10.1186/s13018-020-01684-9. 


\section{Abstract}

Background: Osteoporosis is a metabolic bone disorder that leads to low bone mass and microstructural deterioration of bone tissue and increases bone fractures. Resveratrol, a natural polyphenol compound, has pleiotropic effects including anti-oxidative, anti-aging, and anti-cancer effects. Resveratrol also has roles in increasing osteogenesis and in up-regulating mitochondrial biogenesis of bone marrow-derived mesenchymal stem cells (BM-MSCs). However, it is still unclear that resveratrol can enhance osteogenic differentiation or mitochondrial biogenesis of periosteum-derived MSCs (PO-MSCs), which play key roles in bone tissue maintenance and fracture healing. Thus, in order to test a possible preventive or therapeutic effect of resveratrol on osteoporosis, this study investigated effects of resveratrol treatments on osteogenic differentiation and mitochondrial biogenesis of PO-MSCs.

Methods: The optimal doses of resveratrol treatment on PO-MSCs were determined by cell proliferation and viability assays. Osteogenic differentiation of PO-MSCs under resveratrol treatment was assessed by alkaline phosphatase activities (ALP, an early biomarker of osteogenesis) as well as by extracellular calcium deposit levels (a late biomarker). Mitochondrial biogenesis during osteogenic differentiation of PO-MSCs was measured by quantifying both mitochondrial mass and mitochondrial DNA (mtDNA) contents.

Results: Resveratrol treatments above $10 \mu \mathrm{M}$ seem to have negative effects on cell proliferation and viability of PO-MSCs. Resveratrol treatment (at $5 \mu \mathrm{M}$ ) on PO-MSCs during osteogenic differentiation increased both ALP activities and calcium deposits compared to untreated control groups, demonstrating an enhancing effect of resveratrol on osteogenesis. In addition, resveratrol treatment (at $5 \mu \mathrm{M}$ ) during osteogenic differentiation of PO-MSCs increased both mitochondrial mass and mtDNA copy numbers, indicating that resveratrol can bolster mitochondrial biogenesis in the process of PO-MSC osteogenic differentiation.

Conclusion: Taken together, the findings of this study describe roles of resveratrol in promoting osteogenesis and mitochondrial biogenesis of human PO-MSCs suggesting a possible application of resveratrol as a supplement for osteoporosis and/or osteoporotic fractures.

\section{Background}

Elderly people occupy the fastest growing segment of populations throughout the world. Accordingly, the age-associated bone diseases such as osteoporosis have been increasing. Due to low mineral density and concomitant change of microarchitecture of the bone tissue, osteoporosis manifests as an increased risk of bone fracture (1). The life time risk for an osteoporotic hip, spine, or forearm fracture at the age of 50 years has been estimated to be $40 \sim 53 \%$ in women and $13 \sim 22 \%$ in men (2). These osteoporotic fractures are not only problematic in the fracture itself, but they also exacerbate the patient's medical condition, affect mortality, and increase socioeconomic costs $(3,4)$. Therefore, many attempts aimed at diagnosis and treatment for osteoporosis have been conducted to prevent osteoporotic fracture. 
Adult mesenchymal stem cells (MSCs) are multipotent stem cells capable of differentiating into various cell types including adipocyte, chondrocyte and osteocyte (5). In addition, adult MSCs have some benefits regarding immunologic and ethical issues relative to embryonic stem cells. Thus, MSCs have been actively applied as a material for regenerative medicine for osteoporosis treatment (6-8). Traditionally, bone marrow-derived MSCs (BM-MSCs) have been widely chosen as a stem cell source in regenerative medicine (9). However, BM-MSCs also have some limitations. For example, BM-MSC isolation needs bone marrow aspiration which is painful and has a risk of infection. Furthermore, BM-MSCs from older bone marrow were reported to have decreased expansion and differentiation potentials $(10,11)$. Unfortunately, there is a report that BM-MSCs do not make much contribution to fracture healing (12). Besides the bone marrow, the periosteum also contains adult MSCs. These periosteum-derived MSCs (PO-MSCs) are known to play an important role in fracture healing (12). Moreover, PO-MSCs seem to maintain their differentiation potentials even with increasing age $(13,14)$. These advantages make PO-MSCs an attractive MSC source in regenerative medicine for the bone (15).

Recently, metabolic shift from glycolysis to mitochondrial oxidative phosphorylation (OXPHOS) is taking on increased importance in stem cell differentiation $(16,17)$. Glycolytic metabolism is known as the main metabolism for self-renewal and maintenance in proliferating stem cells. However, when stem cells commit to differentiate, glycolytic metabolism is shifted to mitochondrial OXPHOS to meet an increased cellular energy demand in differentiated cells $(18,19)$. OXPHOS occurs within mitochondria where the mitochondrial respiratory complexes and the ATP synthase produce ATP. Catalytic core subunits of mitochondrial respiratory complexes as well as the ATP synthase are encoded in mitochondrial DNA ( $m$ tDNA). Thus, if cells require more ATP during stem cell differentiation, cells need to up-regulate mitochondrial biogenesis leading to an increased cellular mitochondrial mass and mtDNA contents.

Resveratrol is a natural polyphenol found in red grapes, peanuts, berries, and pomegranates etc $(20,21)$. Resveratrol has pleiotropic effects including anti-oxidative, anti-aging, and anti-cancer effects (22-25). Among these diverse effects of resveratrol, one notable benefit of resveratrol is to increase mitochondrial biogenesis in mammalian cells $(26,27)$. Moreover, resveratrol also promotes osteogenic differentiation of bone marrow or adipose tissue derived mesenchymal stem cells $(28,29)$. However, so far, it is still unclear that resveratrol can enhance osteogenic differentiation or mitochondrial biogenesis of PO-MSCs which play important roles in bone fracture healing. Thus, this study investigated whether resveratrol has a role in osteogenic differentiation and mitochondrial biogenesis of PO-MSCs.

\section{Materials And Methods}

\section{Reagents, in vitro cell cultures, and osteogenesis of human periosteum-derived mesenchymal stem cells (PO-MSCs)}

All chemicals used in this study were purchased from Sigma-Aldrich (St. Louis, MO, USA). Cell culture media and fetal bovine serum were purchased from Invitrogen (Waltham, MA, USA). Human periosteal tissues were obtained from patients who granted informed consent for collection of the tissues, as 
required by the Ethics Committee of Gyeongsang National University Hospital (GNUH 2014-05-012). POMSCs were then isolated as described previously (19). Briefly, periosteal explants were harvested from mandibles during surgical extraction of impacted lower third molars. Periosteal pieces were washed and cultured at $37^{\circ} \mathrm{C}, 95 \%$ humidified air, and $5 \% \mathrm{CO}_{2}$ in 100-mm culture dishes containing Dulbecco's Modified Eagle's Medium (DMEM) supplemented with 10\% heat-inactivated fetal bovine serum, 100 $\mathrm{IU} / \mathrm{mL}$ penicillin, and $100 \mu \mathrm{g} / \mathrm{mL}$ streptomycin. Resulting adherent cells were passaged by gentle trypsinization and reseeding in a fresh medium. The cell culture medium was changed every 3 days during the isolation period. For osteogenic differentiation, PO-MSCs were cultured using osteogenesis induction medium (OM) which is composed of DMEM, supplemented with $50 \mu \mathrm{g} / \mathrm{mL} \mathrm{L-ascorbic} \mathrm{acid} \mathrm{2-}$ phosphate, $10 \mathrm{nM}$ dexamethasone, and $10 \mathrm{mM} \beta$-glycerophosphate (19).

\section{Assessment of PO-MSC proliferation and viability under resveratrol treatments}

Proliferation of PO-MSCs was measured by a cell counting. Briefly, $2 \times 10^{4}$ cells were seeded in 24 -well plates and were cultured in $\mathrm{OM}$ medium. Resveratrol treatment was performed by treating cells with vehicle (ethanol) or a range of resveratrol concentrations from $500 \mathrm{nM}$ upto $20 \mu \mathrm{M}$. 5 day and 10 day cultures were trypsinized and resulting detached cells were counted with a hemocytomer. Viability of POMSCs treated with resveratrol was determined by MTT assays. Shortly, $2 \times 10^{4}$ cells were seeded in 24well plates and cultured in $\mathrm{OM}$ medium with resveratrol treatments ( $500 \mathrm{nM} \sim 20 \mu \mathrm{M}) .5$ day and 10 day cultures were subjected to a colorimetric MTT assay.

\section{Measurements of alkaline phosphatase (ALP) activities}

ALP activities were determined colorimetrically using an ALP Assay Kit (Takara, Kusatsu, Japan) according to the manufacturer's instructions. In brief, whole cell lysates were prepared using a NP-40 lysis buffer (Thermo Scientific, Waltham, USA). Cell lysates were then incubated with p-nitrophenylphosphate, a colorless substrate for ALP, in a Tris-HCl buffer $\left(\mathrm{pH} \mathrm{9.5)}\right.$ at $37^{\circ} \mathrm{C}$ for $15 \mathrm{~min}$. Upon splitting off the phosphate group of $p$-nitrophenylphosphate by ALP, $p$-nitrophenol is released and detected spectrophotometrically (absorption maximum, $405 \mathrm{~nm}$ ). These ALP activities then were normalized to cellular protein contents determined by the Bradford method.

\section{Measurements of calcium deposits}

Mineralization of PO-MSC cultures with resveratrol treatments (500 nM and $5 \mu \mathrm{M}$ ) in OM medium was assessed by von Kossa staining [30]. Calcium contents during osteogenesis of PO-MSCs were also measured by a calcium deposition assay. In brief, at days 14 and 21 of cell cultures, PO-MSCs grown in $\mathrm{OM}$ medium with resveratrol treatments $(500 \mathrm{nM}$ and $5 \mu \mathrm{M})$ were decalcified with $0.6-\mathrm{N} \mathrm{HCl}$ for 1 day at room temperature. Then, the calcium content was determined by the colorimetrical o-cresolphthalein complexone method (Calcium C-test, Wako Chemicals, Japan), whereby calcium reacts with ocresolphthalein to form a purple complex that absorbs light with wavelength of $570 \mathrm{~nm}$. After decalcification, the total protein content in the supernatants was measured by the Bradford method and was used to normalize calcium content. 


\section{Measurements of mitochondrial mass by flow cytometry and fluorescent microscopy}

Mitochondrial mass of cultured PO-MSCs were quantified by flow cytometry. Briefly, $1 \times 10^{5}$ cells were stained in PBS with MitoTracker Green FM dye (Invitrogen, Waltham, MA, USA) for 30 min, washed, and resuspended in $200 \mu \mathrm{l}$ PBS with $1 \%$ fetal bovine serum. Cellular fluorescence signals were then analyzed using a FACSCalibur (BD Biosciences, San Jose, CA, USA). Resulting flow cytometry data were analyzed using FlowJo software version 8.7.3 (Ashland, OR, USA). For fluorescent microcopy, PO-MSCs cultured on chamber slides were stained with MitoTracker Green FM dye. Cellular mitochondria were visualized under a fluorescent microscope (Zeiss Axio Observer Z1, Carl Zeiss, Oberkochen, Germany) and images were analyzed using Image J software ( $\mathrm{NIH}$, Bethesda, $\mathrm{CA}$, USA).

\section{mtDNA copy number analysis by quantitative PCR}

For mtDNA copy number analysis, total cellular DNA was extracted from cultured PO-MSCs. A quantitative real-time PCR method was used to determine the relative abundance of mtDNA versus nuclear 18S rDNA using corresponding mitochondrial and nuclear PCR primer sets in two parallel PCR reactions as described previously (19). Relative mtDNA copy number was calculated as the ratio of the amount of amplification obtained with mtDNA versus nuclear 18S rDNA PCR primer sets for each sample and plotted normalized to the control group. The sequence of the PCR primer pairs are as follows: the nuclear $18 \mathrm{~S}$ rRNA fragment was amplified by the primer pair 5'-TAGAGGGACAAGTGGCGTTC-3' and 5'CGCTGAGCCAGTCAGTGT-3'; and the mitochondrial COX1 fragment was amplified by the primer pair 5'CACCCAAGAACAGGg TTTGT-3-3' and 5 '-TGgCCATGGGTATGTTGTTAA-3'.

\section{Statistical analysis}

All experiments were performed using at least three independent cell cultures. Error bars in all figures represent the mean \pm SEM and statistical analyses were computed using Graphpad Prism 7 software (GraphPad, San Diego, CA, USA). The Student's two-tailed t-test was used for the determination of statistical significance between groups, and a $p$ value of $<0.05$ was considered statistically significant.

\section{Results}

\section{Resveratrol treatments affect neither PO-MSC proliferation nor its viability.}

In order to see effects of resveratrol on cell proliferation and viability during osteogenesis, PO-MSCs were cultured in OM medium for 5 and 10 days. For the same periods of cultures, these PO-MSCs were also treated with various concentrations of resveratrol from $500 \mathrm{nM}$ upto $20 \mu \mathrm{M}$. At day 5 and day 10 cultures, cells were harvested and counted with a hemocytometer for cell proliferation or were subjected to an MTT assay for cell viability. As shown in Fig. 1A $500 \mathrm{nM}, 1 \mu \mathrm{M}$, and $5 \mu \mathrm{M}$ resveratrol treatments does not affect PO-MSC proliferation for 5 and 10 days of osteogenic cultures compared to untreated or vehicle (ethanol) controls. However, $10 \mu \mathrm{M}$ and $20 \mu \mathrm{M}$ resveratrol treatments for 10 days decreased PO-MSC proliferation by about $30 \%$ relative to controls. Similarly, from Fig. 1B, regarding cell viability during 
osteogenic cultures of PO-MSCs, lower concentrations of resveratrol treatments $(500 \mathrm{nM}, 1 \mu \mathrm{M}$, and 5 $\mu \mathrm{M})$ had no effects but higher concentrations of resveratrol (10 $\mu \mathrm{M}$ and $20 \mu \mathrm{M})$ decreased PO-MSC viability by up to $20 \%$ compared to controls. These results indicate that resveratrol treatments below $5 \mu \mathrm{M}$ do not alter PO-MSC proliferation and viability during osteogenic cell cultures for at least 10 days. From these results, two different concentrations of resveratrol ( $500 \mathrm{nM}$ and $5 \mu \mathrm{M})$ were chosen for the subsequent experiments in this study.

\section{Resveratrol treatments increase ALP activities in PO-MSCs during osteogenesis.}

An increase of ALP activity is known as an early biomarker for osteogenic differentiation or osteoblast activity. To test an effect of resveratrol on osteogenic differentiation of PO-MSCs, ALP activities were assessed by a colorimetric assay described in the Methods section. Fig. 2 shows ALP activities of POMSCs triggered to differentiate into osteoblast lineage for 5 and 10 days. Compared to undifferentiated PO-MSCs grown in DMEM, ALP activities were clearly increased in PO-MSCs undergoing osteogenic differentiation by OM culture conditions indicating that OM induces osteogenesis in PO-MSCs. The increase of ALP activity in osteogenically differentiating PO-MSCs is further enhanced by resveratrol treatments ( $500 \mathrm{nM}$ and $5 \mu \mathrm{M})$ in time- and dose-dependent manners relative to untreated controls although $500 \mathrm{nM}$ resveratrol treatment did not show a statistical significance. These results indicate that resveratrol can promote osteogenic differentiation of PO-MSCs.

\section{Resveratrol treatments promote mineralization in osteogenic cultures of PO-MSCs.}

Mineralization by calcium deposits is known as a late biomarker for osteogenesis. In order to test if resveratrol can bolster mineralization during osteogenic differentiation, PO-MSCs were cultured in OM conditions for 2 and 3 weeks with or without resveratrol in culture medium. From von Kossa staining images which mark mineralization shown in Fig. 3A, OM condition led to more mineralization in PO-MSC cultures compared to the control DMEM condition. This mineralization is further increased by resveratrol treatments ( $500 \mathrm{nM}$ and $5 \mu \mathrm{M})$ in time- and dose-dependent manners relative to untreated OM condition. In addition, colorimentric quantitation assays for calcium contents in cell cultures showed that calcium contents in PO-MSCs were increased by OM condition relative to DMEM condition, and this increase were further enhanced by resveratrol treatments (Fig. 3B). Together, these results demonstrate that $500 \mathrm{nM}$ and $5 \mu \mathrm{M}$ resveratrol treatments can enhance matrix mineralization during osteogenic differentiation of POMSCs.

\section{Resveratrol treatments up-regulate mitochondrial biogenesis during the osteogenic differentiation of PO- MSCs.}

Recently, up-regulation of mitochondrial biogenesis has been reported as a characteristic of differentiated MSCs. Moreover, it is well known that resveratrol has a beneficial effect on mitochondrial biogenesis in various cell types. Thus, to test whether mitochondrial biogenesis is up-regulated by resveratrol during osteogenic differentiation of PO-MSCs, mitochondrial mass and mtDNA contents were measured. POMSCs were cultured and induced to osteogenic differentiation by OM with or without resveratrol 
treatments. The resulting differentiated PO-MSCs were stained with a fluorescent dye, Mitotracker Green FM (Invitrogen) which is localized in mitochondria in the cell. The stained cells were then assessed for mitochondrial mass by flow cytometry. From Fig. 4A, compared to undifferentiated DMEM controls, OM increased mitochondrial mass and this increase was further enhanced by resveratrol treatments in timeand dose-dependent manners. In addition, from fluorescent microscopic images at 2 weeks osteogenic cultures of PO-MSCs, resveratrol increased mitochondrial mass in a dose-dependent manner (Fig. 4B). Furthermore, mtDNA contents measured by qPCR were also increased by resveratrol treatments in osteogenically differentiated PO-MSCs relative to controls (Fig. 4C). Together, these findings clearly showed that mitochondrial biogenesis can be up-regulated by resveratrol during the osteogenic differentiation of PO-MSCs.

\section{Discussion}

In this study, we demonstrated that resveratrol treatments $(5 \mu \mathrm{M})$ up-regulate both ALP activities and calcium deposits during osteogenic differentiation of PO-MSCs compared to the untreated controls. These results indicate that resveratrol can enhance osteogenic differentiation of PO-MSCs. In other studies, resveratrol was reported to promote osteogenic differentiation of bone marrow or adipose tissue derived MSCs at higher concentrations (28-32). However, in this study, PO-MSC viability decreased when resveratrol dose was above $10 \mu \mathrm{M}$. It seems that the osteogenic effect of resveratrol varies according to dosage or cell types (33-37). Therefore, further investigation on the optimal dosage and administration method of resveratrol for accelerating osteogenesis of PO-MSC is needed.

In addition, this study showed that resveratrol treatments increase both mitochondrial mass and mtDNA contents during osteogenic differentiation in PO-MSCs. These findings indicate that resveratrol can upregulate mitochondrial biogenesis during osteogenic differentiation of PO-MSCs and are consistent with other reports regarding mitochondrial biogenesis by resveratrol in various cell types (26, 38-42). Moreover, the up-regulation of mitochondrial biogenesis by resveratrol in PO-MSCs is in line with the notion that metabolic shift from glycolysis to mitochondrial OXPHOS occurs during stem cell differentiation $(16,17$, 43-47). Thus, these results suggest that a small molecule up-regulating mitochondrial biogenesis such as resveratrol can be a new modulator that may enhance osteogenesis in adult PO-MSCs for regenerative medicine.

Currently, the most widely used therapeutic agents for osteoporosis are anti-resorptive drugs such as bisphosphonate and selective estrogen receptor modulators (SERMs) (48). However, these medications can cause complications when used for a long time. In particular, bisphosphonate has complications such as gastrointestinal irritation, atypical fracture due to decreased bone replacement rate, and osteonecrosis of jaw bone (49-52). In addition, venous thromboembolism can occur as a complication when using SERMs $(53,54)$. On the other hand, a bone anabolic agent, teriparatide (a parathyroid hormone analog) has recently been used for osteoporosis treatment. But its disadvantages are the high cost, the need for injection, and the possibility of developing malignant tumors over a long period of time (55). Thus, there is an increasing demand for a new bone anabolic agent. Interestingly, recent studies 
have shown that oral intake of adequate amounts of resveratrol can prevent bone fractures $(56,57)$. In this regard, this study suggests that resveratrol supplements may be clinically meaningful in increasing bone tissue formation in osteoporotic bones.

Fracture healing in osteoporotic bone is poor compared to fractures occurring at young age. For example, fixation failure or nonunion rates of osteoporotic fractures were reported to be approximately $15 \sim 24.9 \%$ depending on specific fractures $(58,59)$. Cellular factors contributing to delayed healing can be a decreased number of MSCs and/or osteoblasts with increasing age as well as an impaired response of bone cells in osteoporotic bone (60). Therefore, regenerative medicine utilizing adult stem cells has been spotlighted in the prevention and treatment of osteoporosis and osteoporotic fracture $(6-8,58,59)$. However, BM-MSCs have some disadvantages, for example, a decreased cellular expansion and differentiation potentials from older bone marrow tissues. These limitations hinder BM-MSCs from their therapeutic application (10-12). Alternatively, PO-MSCs derived from the periosteum of the bone have some benefits. Because they locate in the periosteum, it is expected that they are easy to participate in bone tissue maintenance in vivo. Moreover, the expansion and differentiation potentials of PO-MSCs are maintained even in older ages $(13,14)$. Thus, PO-MSCs were expected to play a more critical role in bone healing than BM-MSCs $(12,63)$. Recently, several studies have found that sclerostin antibody has clinical implications for the treatment of osteoporosis $(64,65)$. Thompson et al. $(66)$ reported that systemic administration of sclerostin antibody in geriatric mice can enhance the proliferation of osteogenic cells in the periosteum of geriatric mice and can contribute to an increase in cortical bone thickness. Thus, the periosteum of the elderly may be an important target tissue for fracture healing and osteoporosis treatment. Although this study has shown that resveratrol can promote the osteogenesis of MSCs derived from the periosteal tissue, there are apparent limitations of its use as a treatment for osteoporosis. For example, because this study has utilized in vitro cell culture systems, a possible bone formation effect of resveratrol should be tested in in vivo models, such as geriatric animals or bone defects. Further studies aimed at an effective dose of resveratrol for in vivo animal models will also be needed for its clinical applicability and efficacy. In summary, promoting osteogeneis of PO-MSCs by a small molecule such as resveratrol can be clinically valuable for the treatment of osteoporosis and accompanying osteoporotic fracture. This study demonstrates that resveratrol, a well-known small molecule up-regulating mitochondrial biogenesis can be used as a new modulator for osteogenic differentiation of PO-MSCs. Thus, this study provides a new experimental paradigm to investigate the osteogenesis of adult stem cells and the role of mitochondrial biogenesis in regenerative medicine for the bone.

\section{Conclusion}

In conclusion, this study demonstrates that resveratrol promotes osteogenic differentiation and mitochondrial biogenesis of PO-MSCs. Therefore, enhancing osteogenesis of PO-MSCs by small molecules such as resveratrol may be clinically valuable for the treatment of osteoporosis and accompanying osteoporotic fracture. 


\section{Abbreviations}

ALP: alkaline phosphatase; BM-MSC: bone marrow-derived mesenchymal stem cell; DMEM: Dulbecco's modified eagle medium; OM: osteogenesis induction medium; PO-MSC: periosteum-derived mesenchymal stem cells

\section{Declarations}

\section{Funding}

This work was supported by the National Research Foundation of Korea (Grants \#: NRF2019R1F1060013) and by the Gyeongsang National University Hospital of Korea (biomedical research institute fund \#: GNUHBIF-2015-0010).

\section{Availability of data and materials}

The materials described in the manuscript will be available to all scientists for non-commercial purposes.

\section{Ethics approval and consent to participate}

This study was approved by the Ethics Committee of Gyeongsang National University Hospital (GNUH 2014-05-012) of Korea. The patients had given their consent to participate in this study.

\section{Consent for publication}

Not applicable.

\section{Competing interests}

The authors declare that they have no competing interests.

\section{Author details}

${ }^{1}$ Department of Orthopedic Surgery and Institute of Health Sciences, Gyeongsang National University, school of medicine and Gyeongsang National University Hospital, Jinju, Republic of Korea. ${ }^{2}$ College of Pharmacy and Research Institute of Pharmaceutical Sciences, Gyeongsang National University, Jinju, Republic of Korea. ${ }^{3}$ Department of Theriogenology and Biotechnology, College of Veterinary Medicine, Gyeongsang National University, Jinju, Republic of Korea. ${ }^{4}$ Department of Oral and Maxillofacial Surgery and Institute of Health Sciences, School of Medicine and Hospital, Gyeongsang National University, Jinju, Republic of Korea

\section{References}

1. Osteoporosis prevention, diagnosis, and therapy. NIH Consens Statement. 2000;17(1):1-45. 
2. Johnell O, Kanis J. Epidemiology of osteoporotic fractures. Osteoporos Int. 2005;16 Suppl 2:S3-7.

3. Colon-Emeric CS, Saag KG. Osteoporotic fractures in older adults. Best Pract Res Clin Rheumatol. 2006;20(4):695-706.

4. Magaziner J, Chiles N, Orwig D. Recovery after Hip Fracture: Interventions and Their Timing to Address Deficits and Desired Outcomes-Evidence from the Baltimore Hip Studies. Nestle Nutr Inst Workshop Ser. 2015;83:71-81.

5. Dominici M, Le Blanc K, Mueller I, Slaper-Cortenbach I, Marini F, Krause D, et al. Minimal criteria for defining multipotent mesenchymal stromal cells. The International Society for Cellular Therapy position statement. Cytotherapy. 2006;8(4):315-7.

6. Ye X, Zhang P, Xue S, Xu Y, Tan J, Liu G. Adipose-derived stem cells alleviate osteoporosis by enhancing osteogenesis and inhibiting adipogenesis in a rabbit model. Cytotherapy. 2014;16(12):1643-55.

7. Mirsaidi A, Genelin K, Vetsch JR, Stanger S, Theiss F, Lindtner RA, et al. Therapeutic potential of adipose-derived stromal cells in age-related osteoporosis. Biomaterials. 2014;35(26):7326-35.

8. Oryan A, Kamali A, Moshiri A, Baghaban Eslaminejad M. Role of Mesenchymal Stem Cells in Bone Regenerative Medicine: What Is the Evidence? Cells Tissues Organs. 2017;204(2):59-83.

9. Jin YZ, Lee JH. Mesenchymal Stem Cell Therapy for Bone Regeneration. Clin Orthop Surg. 2018;10(3):271-8.

10. Choudhery MS, Badowski M, Muise A, Pierce J, Harris DT. Donor age negatively impacts adipose tissue-derived mesenchymal stem cell expansion and differentiation. J Transl Med. 2014;12:8.

11. Berebichez-Fridman R, Gomez-Garcia R, Granados-Montiel J, Berebichez-Fastlicht E, Olivos-Meza A, Granados J, et al. The Holy Grail of Orthopedic Surgery: Mesenchymal Stem Cells-Their Current Uses and Potential Applications. Stem Cells Int. 2017;2017:2638305.

12. Colnot C. Skeletal cell fate decisions within periosteum and bone marrow during bone regeneration. $J$ Bone Miner Res. 2009;24(2):274-82.

13. De Bari C, Dell'Accio F, Luyten FP. Human periosteum-derived cells maintain phenotypic stability and chondrogenic potential throughout expansion regardless of donor age. Arthritis Rheum. 2001;44(1):85-95.

14. De Bari C, Dell'Accio F, Vanlauwe J, Eyckmans J, Khan IM, Archer CW, et al. Mesenchymal multipotency of adult human periosteal cells demonstrated by single-cell lineage analysis. Arthritis Rheum. 2006;54(4):1209-21.

15. Breitbart AS, Grande DA, Kessler R, Ryaby JT, Fitzsimmons RJ, Grant RT. Tissue engineered bone repair of calvarial defects using cultured periosteal cells. Plast Reconstr Surg. 1998;101(3):567-74; discussion 75-6.

16. Chen CT, Shih YR, Kuo TK, Lee OK, Wei YH. Coordinated changes of mitochondrial biogenesis and antioxidant enzymes during osteogenic differentiation of human mesenchymal stem cells. Stem Cells. 2008;26(4):960-8. 
17. Zhang Y, Marsboom G, Toth PT, Rehman J. Mitochondrial respiration regulates adipogenic differentiation of human mesenchymal stem cells. PLoS One. 2013;8(10):e77077.

18. Pattappa G, Heywood HK, de Bruijn JD, Lee DA. The metabolism of human mesenchymal stem cells during proliferation and differentiation. J Cell Physiol. 2011;226(10):2562-70.

19. Lee AR, Moon DK, Siregar A, Moon SY, Jeon RH, Son YB, et al. Involvement of mitochondrial biogenesis during the differentiation of human periosteum-derived mesenchymal stem cells into adipocytes, chondrocytes and osteocytes. Arch Pharm Res. 2019;42(12):1052-62.

20. Burns J, Yokota T, Ashihara H, Lean ME, Crozier A. Plant foods and herbal sources of resveratrol. J Agric Food Chem. 2002;50(11):3337-40.

21. Sales JM, Resurreccion AV. Resveratrol in peanuts. Crit Rev Food Sci Nutr. 2014;54(6):734-70.

22. Truong VL, Jun M, Jeong WS. Role of resveratrol in regulation of cellular defense systems against oxidative stress. Biofactors. 2018;44(1):36-49.

23. Li YR, Li S, Lin CC. Effect of resveratrol and pterostilbene on aging and longevity. Biofactors. 2018;44(1):69-82.

24. Varoni EM, Lo Faro AF, Sharifi-Rad J, Iriti M. Anticancer Molecular Mechanisms of Resveratrol. Front Nutr. 2016;3:8.

25. Kim CW, Hwang KA, Choi KC. Anti-metastatic potential of resveratrol and its metabolites by the inhibition of epithelial-mesenchymal transition, migration, and invasion of malignant cancer cells. Phytomedicine. 2016;23(14):1787-96.

26. Csiszar A, Labinskyy N, Pinto JT, Ballabh P, Zhang H, Losonczy G, et al. Resveratrol induces mitochondrial biogenesis in endothelial cells. Am J Physiol Heart Circ Physiol. 2009;297(1):H13-20.

27. Zheng J, Chen LL, Zhang HH, Hu X, Kong W, Hu D. Resveratrol improves insulin resistance of catchup growth by increasing mitochondrial complexes and antioxidant function in skeletal muscle. Metabolism. 2012;61(7):954-65.

28. Li J, Xin Z, Cai M. The role of resveratrol in bone marrow-derived mesenchymal stem cells from patients with osteoporosis. J Cell Biochem. 2019;120(10):16634-42.

29. Erdman CP, Dosier CR, Olivares-Navarrete R, Baile C, Guldberg RE, Schwartz Z, et al. Effects of resveratrol on enrichment of adipose-derived stem cells and their differentiation to osteoblasts in two-and three-dimensional cultures. J Tissue Eng Regen Med. 2012;6 Suppl 3:s34-46.

30. Zhou H, Shang L, Li X, Zhang X, Gao G, Guo C, et al. Resveratrol augments the canonical Wnt signaling pathway in promoting osteoblastic differentiation of multipotent mesenchymal cells. Exp Cell Res. 2009;315(17):2953-62.

31. Shakibaei M, Shayan P, Busch F, Aldinger C, Buhrmann C, Lueders C, et al. Resveratrol mediated modulation of Sirt-1/Runx2 promotes osteogenic differentiation of mesenchymal stem cells: potential role of Runx2 deacetylation. PLoS One. 2012;7(4):e35712.

32. Huang Y, Huo J, Liu FQ, Liu J, Zhang XJ, Guo CH, et al. Resveratrol Promotes in vitro Differentiation of Osteoblastic MC3T3-E1 Cells via Potentiation of the Calcineurin/NFATc1 Signaling Pathway. 
Biochemistry (Mosc). 2019;84(6):686-92.

33. Posadino AM, Giordo R, Cossu A, Nasrallah GK, Shaito A, Abou-Saleh H, et al. Flavin Oxidase-Induced ROS Generation Modulates PKC Biphasic Effect of Resveratrol on Endothelial Cell Survival. Biomolecules. 2019;9(6).

34. Guo X, Ni J, Dai X, Zhou T, Yang G, Xue J, et al. Biphasic regulation of spindle assembly checkpoint by low and high concentrations of resveratrol leads to the opposite effect on chromosomal instability. Mutat Res Genet Toxicol Environ Mutagen. 2018;825:19-30.

35. San Hipolito-Luengo A, Alcaide A, Ramos-Gonzalez M, Cercas E, Vallejo S, Romero A, et al. Dual Effects of Resveratrol on Cell Death and Proliferation of Colon Cancer Cells. Nutr Cancer. 2017;69(7):1019-27.

36. Li Q, Huyan T, Ye LJ, Li J, Shi JL, Huang QS. Concentration-dependent biphasic effects of resveratrol on human natural killer cells in vitro. J Agric Food Chem. 2014;62(45):10928-35.

37. Peltz L, Gomez J, Marquez M, Alencastro F, Atashpanjeh N, Quang T, et al. Resveratrol exerts dosage and duration dependent effect on human mesenchymal stem cell development. PLoS One. 2012;7(5):e37162.

38. Ferretta A, Gaballo A, Tanzarella P, Piccoli C, Capitanio N, Nico B, et al. Effect of resveratrol on mitochondrial function: implications in parkin-associated familiar Parkinson's disease. Biochim Biophys Acta. 2014;1842(7):902-15.

39. Kim SK, Joe Y, Zheng M, Kim HJ, Yu JK, Cho GJ, et al. Resveratrol induces hepatic mitochondrial biogenesis through the sequential activation of nitric oxide and carbon monoxide production. Antioxid Redox Signal. 2014;20(16):2589-605.

40. Peng K, Tao Y, Zhang J, Wang J, Ye F, Dan G, et al. Resveratrol Regulates Mitochondrial Biogenesis and Fission/Fusion to Attenuate Rotenone-Induced Neurotoxicity. Oxid Med Cell Longev. 2016;2016:6705621.

41. Cao MM, Lu X, Liu GD, Su Y, Li YB, Zhou J. Resveratrol attenuates type 2 diabetes mellitus by mediating mitochondrial biogenesis and lipid metabolism via Sirtuin type 1. Exp Ther Med. 2018;15(1):576-84.

42. Zhang H, Li Y, Su W, Ying Z, Zhou L, Zhang L, et al. Resveratrol attenuates mitochondrial dysfunction in the liver of intrauterine growth retarded suckling piglets by improving mitochondrial biogenesis and redox status. Mol Nutr Food Res. 2017;61(5).

43. Varum S, Rodrigues AS, Moura MB, Momcilovic O, Easley CAt, Ramalho-Santos J, et al. Energy metabolism in human pluripotent stem cells and their differentiated counterparts. PLoS One. 2011;6(6):e20914.

44. Hofmann AD, Beyer M, Krause-Buchholz U, Wobus M, Bornhauser M, Rodel G. OXPHOS supercomplexes as a hallmark of the mitochondrial phenotype of adipogenic differentiated human MSCs. PLoS One. 2012;7(4):e35160.

45. Pietila M, Palomaki S, Lehtonen S, Ritamo I, Valmu L, Nystedt J, et al. Mitochondrial function and energy metabolism in umbilical cord blood- and bone marrow-derived mesenchymal stem cells. Stem 
Cells Dev. 2012;21(4):575-88.

46. Ito K, Suda T. Metabolic requirements for the maintenance of self-renewing stem cells. Nat Rev Mol Cell Biol. 2014;15(4):243-56.

47. Shum LC, White NS, Mills BN, Bentley KL, Eliseev RA. Energy Metabolism in Mesenchymal Stem Cells During Osteogenic Differentiation. Stem Cells Dev. 2016;25(2):114-22.

48. Cosman F, de Beur SJ, LeBoff MS, Lewiecki EM, Tanner B, Randall S, et al. Clinician's Guide to Prevention and Treatment of Osteoporosis. Osteoporos Int. 2014;25(10):2359-81.

49. Khan AA, Morrison A, Hanley DA, Felsenberg D, McCauley LK, O'Ryan F, et al. Diagnosis and management of osteonecrosis of the jaw: a systematic review and international consensus. J Bone Miner Res. 2015;30(1):3-23.

50. Ralston SH, Kou TD, Wick-Urban B, Steinbuch M, Masud T. Risk of upper gastrointestinal tract events in risedronate users switched to alendronate. Calcif Tissue Int. 2010;87(4):298-304.

51. Schilcher J, Koeppen V, Aspenberg P, Michaelsson K. Risk of atypical femoral fracture during and after bisphosphonate use. Acta Orthop. 2015;86(1):100-7.

52. Oh BH, Heo YM, Yi JW, Kim TG, Lee JS. Atypical Fracture of the Proximal Shaft of the Ulna Associated with Prolonged Bisphosphonate Therapy. Clin Orthop Surg. 2018;10(3):389-92.

53. Grady D, Ettinger B, Moscarelli E, Plouffe L, Jr., Sarkar S, Ciaccia A, et al. Safety and adverse effects associated with raloxifene: multiple outcomes of raloxifene evaluation. Obstet Gynecol. 2004;104(4):837-44.

54. Barrett-Connor E, Mosca L, Collins P, Geiger MJ, Grady D, Kornitzer M, et al. Effects of raloxifene on cardiovascular events and breast cancer in postmenopausal women. N Engl J Med. 2006;355(2):125-37.

55. Vahle JL, Sato M, Long GG, Young JK, Francis PC, Engelhardt JA, et al. Skeletal changes in rats given daily subcutaneous injections of recombinant human parathyroid hormone (1-34) for 2 years and relevance to human safety. Toxicol Pathol. 2002;30(3):312-21.

56. Bo S, Gambino R, Ponzo V, Cioffi I, Goitre I, Evangelista A, et al. Effects of resveratrol on bone health in type 2 diabetic patients. A double-blind randomized-controlled trial. Nutr Diabetes. 2018;8(1):51.

57. Wang HD, Shi YM, Li L, Guo JD, Zhang YP, Hou SX. Treatment with resveratrol attenuates sublesional bone loss in spinal cord-injured rats. Br J Pharmacol. 2013;170(4):796-806.

58. Giannoudis PV, Schneider E. Principles of fixation of osteoporotic fractures. J Bone Joint Surg Br. 2006;88(10):1272-8.

59. Parker MJ, Raghavan R, Gurusamy K. Incidence of fracture-healing complications after femoral neck fractures. Clin Orthop Relat Res. 2007;458:175-9

60. Giannoudis P, Tzioupis C, Almalki T, Buckley R. Fracture healing in osteoporotic fractures: is it really different? A basic science perspective. Injury. 2007;38 Suppl 1:S90-9.

61. Wei FY, Chow SK, Leung KS, Qin J, Guo A, Yu OL, et al. Low-magnitude high-frequency vibration enhanced mesenchymal stem cell recruitment in osteoporotic fracture healing through the SDF- 
1/CXCR4 pathway. Eur Cell Mater. 2016;31:341-54.

62. Schafer R, Spohn G, Baer PC. Mesenchymal Stem/Stromal Cells in Regenerative Medicine: Can Preconditioning Strategies Improve Therapeutic Efficacy? Transfus Med Hemother. 2016;43(4):25667.

63. Duchamp de Lageneste O, Julien A, Abou-Khalil R, Frangi G, Carvalho C, Cagnard N, et al. Periosteum contains skeletal stem cells with high bone regenerative potential controlled by Periostin. Nat Commun. 2018;9(1):773.

64. Shakeri A, Adanty C. Romosozumab (sclerostin monoclonal antibody) for the treatment of osteoporosis in postmenopausal women: A review. J Popul Ther Clin Pharmacol. 2020;27(1):e25e31.

65. Mariscal G, Nunez JH, Bhatia S, Barrios C, Domenech-Fernandez P. Safety of Romosozumab in Osteoporotic Men and Postmenopausal Woman: A Meta-Analysis and Systematic Review. Monoclon Antib Immunodiagn Immunother. 2020.

66. Thompson ML, Chartier SR, Mitchell SA, Mantyh PW. Preventing painful age-related bone fractures: Anti-sclerostin therapy builds cortical bone and increases the proliferation of osteogenic cells in the periosteum of the geriatric mouse femur. Mol Pain. 2016;12.

\section{Figures}


A

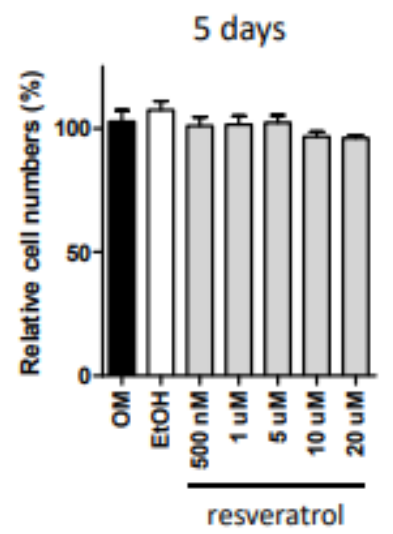

10 days

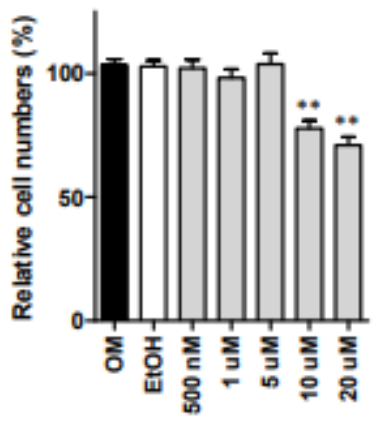

B

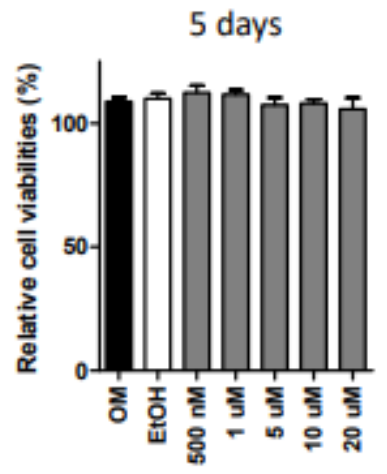

10 days

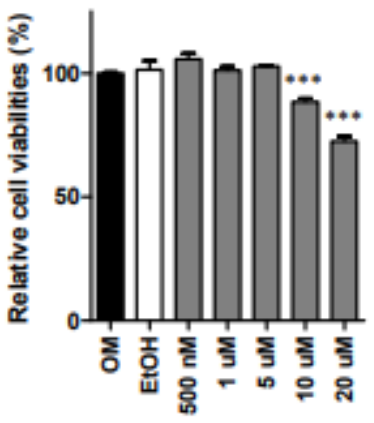

Figure 1

Effects of resveratrol on PO-MSC proliferation and viability. (A) Cell proliferation of PO-MSCs treated with resveratrol under $\mathrm{OM}$ medium for 5 and 10 days was measured by cell counting. (B) Cell viability of POMSCs treated with resveratrol under OM medium for 5 and 10 days was measured by MTT assay.

A

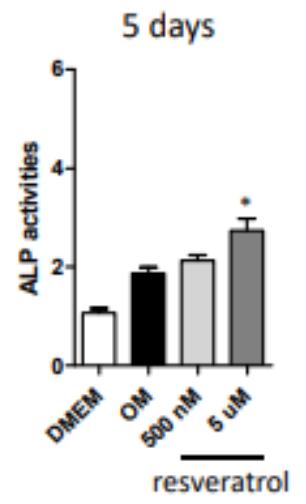

B

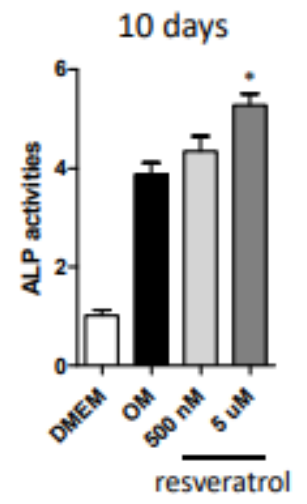

Figure 2 
Effects of resveratrol on ALP activity in PO-MSCs during osteogenesis. ALP activity with resveratrol treatments ( $500 \mathrm{nM}$ and $5 \mu \mathrm{M})$ for 5 and 10 days in OM medium was measured.

A

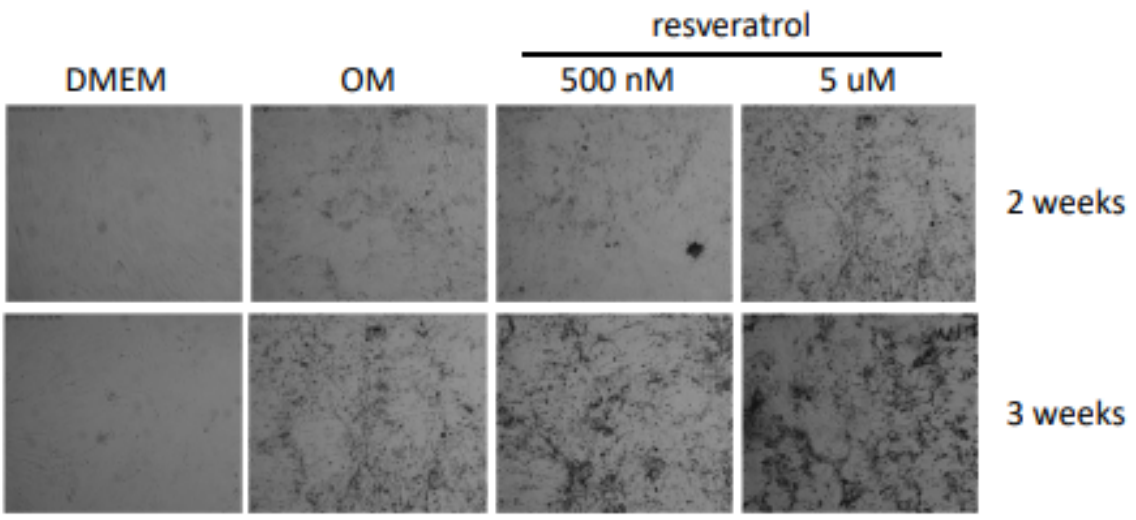

B

2 weeks

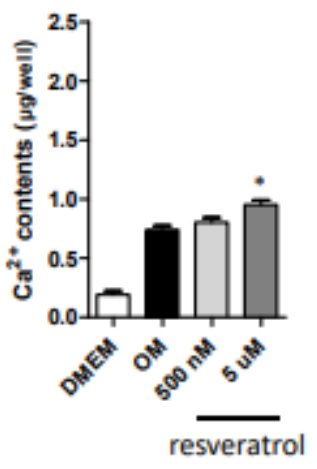

3 weeks

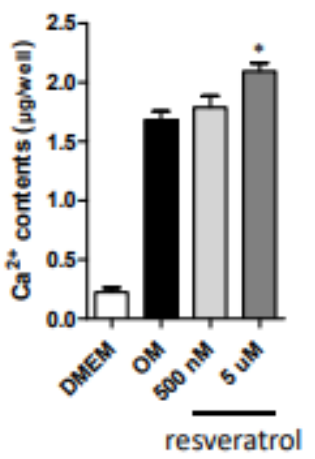

\section{Figure 3}

Effects of resveratrol on calcium deposit levels of PO-MSCs during osteogenesis. (A) Von Kossa staining of PO-MSCs at 2 and 3 weeks of osteogenic cultures. (B) Calcium contents ( $\mu \mathrm{g} / \mathrm{well}$ ) in osteogenic cultures ( 2 and 3 weeks) of PO-MSCs determined by a chromogenic assay. 
A

C
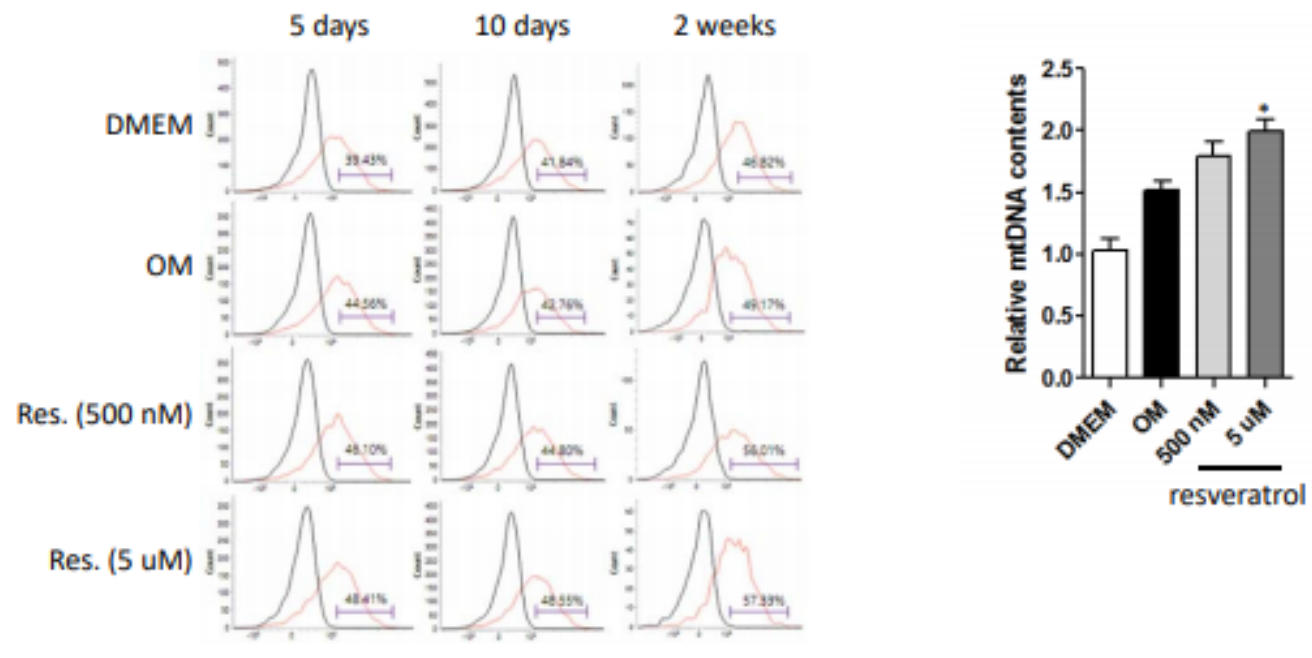

B

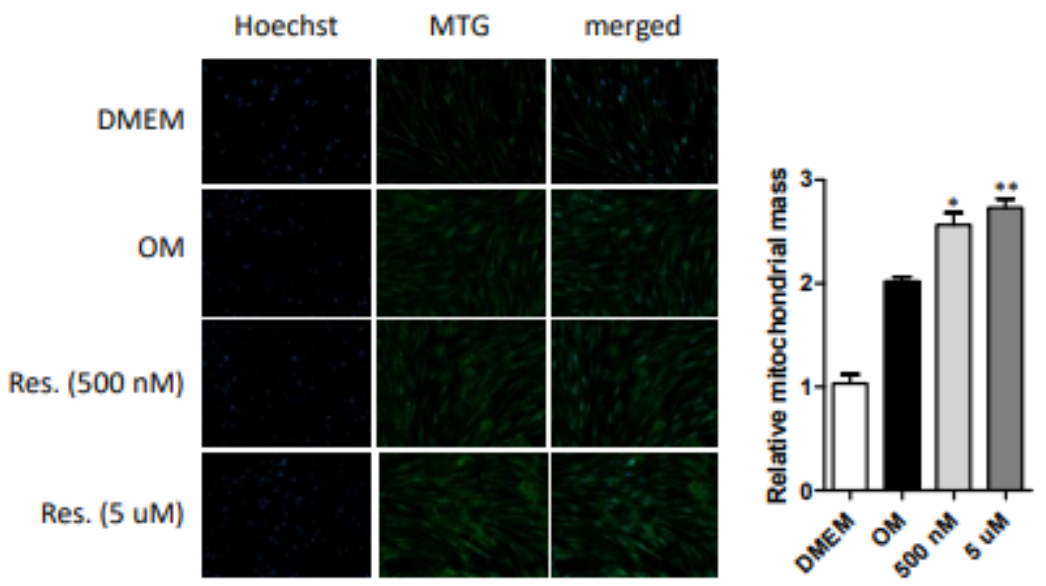

\section{Figure 4}

Effects of resveratrol on mitochondrial biogenesis in PO-MSCs during osteogenesis. (A) Analysis of mitochondrial mass by flow cytometry (black line: unstatined; red line: Mitotracker Green FM (MTG) stained). (B) Fluorescence microscopy of PO-MSCs at 2 weeks of osteogenic cultures using MTG staining and quantification. (C) mtDNA contents determined by qPCR. 